\title{
The Relative Stability of Indole Isomers is a Consequence of the Glidewell-Lloyd Rule
}

\author{
Ricardo Pino-Rios ${ }^{\mathrm{a} *}$, Miquel Solàb*

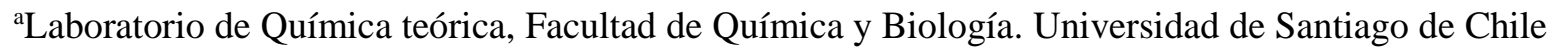 \\ (USACH), Región Metropolitana, Chile; ricardo.pino@usach.cl \\ bInstitute of Computational Chemistry and Catalysis, University of Girona, 17003 Girona, Catalonia, \\ Spain; miquel.sola@udg.edu
}

\begin{abstract}
Indole (1) is a heterocyclic aromatic compound consisting of a pyrrole ring (5MR) fused with a benzene ring (6MR). This compound is highly stable, found in several natural products, and used as a building block for the synthesis of novel organic compounds. On the other hand, its isomers isoindole (2) and indolizine (3) are much less stable and are normally isolated when bonded to other stable compounds. The stability of these compounds has been analyzed in terms of local aromaticity using magnetic, geometric, and delocalization criteria. All criteria used indicate that there is a continuing reduction in aromaticity of the $6 \mathrm{MR}$ whereas, for the 5MR, the aromaticity increases when going from $\mathbf{1}$ to $\mathbf{3}$. This is confirmed by Natural Resonance theory calculations indicating that the resonant structures which retain the aromaticity of $5 \mathrm{MR}$ are the ones having the largest contribution. The results obtained suggest that the relative stability of indole isomers is a consequence of the Glidewell-Lloyd rule.
\end{abstract}

\section{Introduction}

Indole (1) is a highly stable heterocyclic structure consisting of a pyrrolic ring fused (5MR) with a six-membered ring (6MR). This compound is used as building block for the synthesis of new organic compounds and natural products. ${ }^{1-2}$ On the other hand, its isomers, isoindole (2) and indolizine (3) 
are compounds that show a high reactivity and, in fact, in the case of $\mathbf{2}$, its synthesis has taken almost 80 years. It is possible to find systems that contain both isomers, however they generally appear attached to other stable rings or in their respective hydrogenated forms..$^{3-4}$

Previous studies concerning the aromaticity of $\mathbf{2}$ indicate that this compound is considerably less

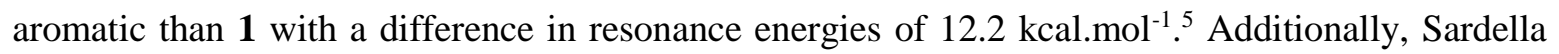
and coworkers ${ }^{6}$ suggest that 2 can be best understood in terms of the interaction between pyrrole ring and 1,3-cis-butadiene in $\mathrm{C} 3$ and $\mathrm{C} 4$ carbons (see 5MR ring numeration in Fig. 1). These results coincide with the work by Mandado et al., ${ }^{7}$ who systematically evaluated the aromaticity of azines, noting that the aromaticity of the 6MR in $\mathbf{1}$ is greater than that of $\mathbf{2}$ and finding the opposite case for the 5MR. In the case of $\mathbf{3}$, no studies have been reported regarding its aromaticity.

The assessment of aromaticity in local rings of non-benzenoid polycyclic aromatic compounds escapes the widely known Clar $\pi$-sextet rule ${ }^{8-9}$ which is applicable to compounds containing only 6MRs. Some years ago, Glidewell and Lloyd generalized the rule proposed by Clar about 50 years ago, currently known as the Glidewell-Lloyd rule $^{10}$ (GLR), which establishes that the population of $\pi$-electrons in conjugated polycyclic systems tends to form the smallest $4 n+2$ groups and to avoid the formation of the smallest $4 n$ groups. This less known rule has recently been applied to an extensive series of compounds in which 4, 6, and 8-membered rings are included to assess their validity. ${ }^{11}$

In the present article, the local aromaticity in $\mathbf{1}$ and its isomers has been assessed by means of a number of aromaticity criteria based on different magnetic, geometric, energetic, and delocalization properties, as usually recommended. ${ }^{12-13}$ The results obtained show that there is a reduction in the aromatic character in the $6 \mathrm{MR}$, whereas the $5 \mathrm{MR}$ presents a slight increase in its aromatic character when the compounds are ordered as follows: $\mathbf{1} \rightarrow \mathbf{2} \rightarrow \mathbf{3}$.

In addition, we have evaluated the Kekule structures that best describe the behavior of these compounds through Natural Resonance Theory (NRT) calculations. ${ }^{14-16}$ The resonant structures with 
the highest weight are those that contribute most to the aromaticity of the smaller 5MR, which is a consequence of the GLR.

\section{Computational Methods}

Geometric optimizations have been performed at the PBE0/6-311G**17-19 level of theory using Gaussian 16 computational package ${ }^{20}$. In addition, vibrational frequency calculations were done at the same level to ensure that we obtained a true minimum on the potential energy surface. In order to study the relative stability of the isomers, single point energy calculations have been carried out at the $\operatorname{CCSD}(\mathrm{T})^{21} / 6-311 \mathrm{G}^{* *}$ level using the $\mathrm{PBE} 0 / 6-311 \mathrm{G}^{* *}$ optimized geometries. Additionally, calculations for naphthalene (4) have been carried out in order to provide comparisons with heterocyclic compounds. Magnetic aromaticity descriptors ${ }^{22}$ have been computed employing the Gauge Independent Atomic Orbital (GIAO) method in gas phase. ${ }^{23}$ Magnetically induced current density $^{24}$ (MICD) maps have been obtained to qualitatively visualize the differences in the aromaticity of the local rings. Moreover, ring current strength (RCS) calculations have been calculated by integrating the current flow that passes through the interatomic surfaces according to the Quantum Theory of Atoms in Molecules ${ }^{25}$ (QTAIM) using the AIMAll software. ${ }^{26-29}$ In addition, NICS $\mathrm{zz}$ calculations were also performed at $1 \AA$ above the center of each local ring at the same level. ${ }^{30-32}$ The geometric criterion has been assessed by means of the Harmonic Oscillator Model of Aromaticity ${ }^{33-}$

${ }^{34}$ (HOMA) that consists in the measurement of the bond distance and equalization deviation of the ideal aromatic benzene molecule. The energetic criteria has been evaluated through the calculation of aromatic stabilization energies using the isomerization stabilization energy method ${ }^{35}$ (ISE) which is obtained through the energy difference between the methyl-substituted aromatic ring and its corresponding isomer with a double exocyclic bond (See Figure S3 in SI). Finally, the delocalization criteria $^{36-37}$ have been evaluated using two indexes: the multicenter delocalization index ${ }^{38}$ (MCI) and the para-delocalization index ${ }^{39}$ (PDI). The former measures the number of electrons shared in the 
$6 \mathrm{MR}$ and 5MR, whereas the latter quantifies the number of electrons shared between the para carbon atoms in the 6MR. Geometric and delocalization calculations have been performed using Multiwfn program. ${ }^{40}$ Natural resonance theory (NRT) calculations have been carried out using NBO 6.0 software. ${ }^{41}$ Plots of MICD vector maps and structures for studied compounds have been made with VisIt 3.0.2 $2^{42}$ and Chemcraft ${ }^{43}$ programs, respectively.

\section{Results and Discussion}

Molecular structures of the studied compounds are shown in Fig. 1. As can be seen, the difference between $\mathbf{1}$ and its two isomers is related to the way that $5 \mathrm{MR}$ is fused to $6 \mathrm{MR}$. In the case of $\mathbf{1}$, the $\mathrm{C} 4$ and $\mathrm{C} 5$ atoms of the pyrrolic ring are in the ring junction, while the 2 is fused with the $6 \mathrm{MR}$ by $\mathrm{C} 3$ and $\mathrm{C} 4$ atoms. Indolizine (3) presents a somewhat different fusion since the pyrrolic ring is fused in the $\mathrm{N} 1$ and $\mathrm{C} 2$ atoms. One of the most noticeable consequences of studied isomers are shown in the bond lengths. An increase (reduction) of the conjugation of the 5MR (6MR) can be observed when the systems are ordered from $\mathbf{1}$ to $\mathbf{3}$. These differences cause variations in the stability of the isomers, the values at the $\operatorname{CCSD}(\mathrm{T}) / 6-311 \mathrm{G}^{* *} / / \mathrm{PBE} 0 / 6-311 \mathrm{G}^{* *}$ level show that the most stable isomer is $\mathbf{1}$, while $\mathbf{2}$ and $\mathbf{3}$ are 7.5 and $10.4 \mathrm{kcal}^{\mathrm{mol}} \mathrm{f}^{-1}$ less stable compared to $\mathbf{1}$. The structural differences found translate into changes in the electron distribution of the systems, which can be observed in the MICD maps depicted in Fig. 2. 

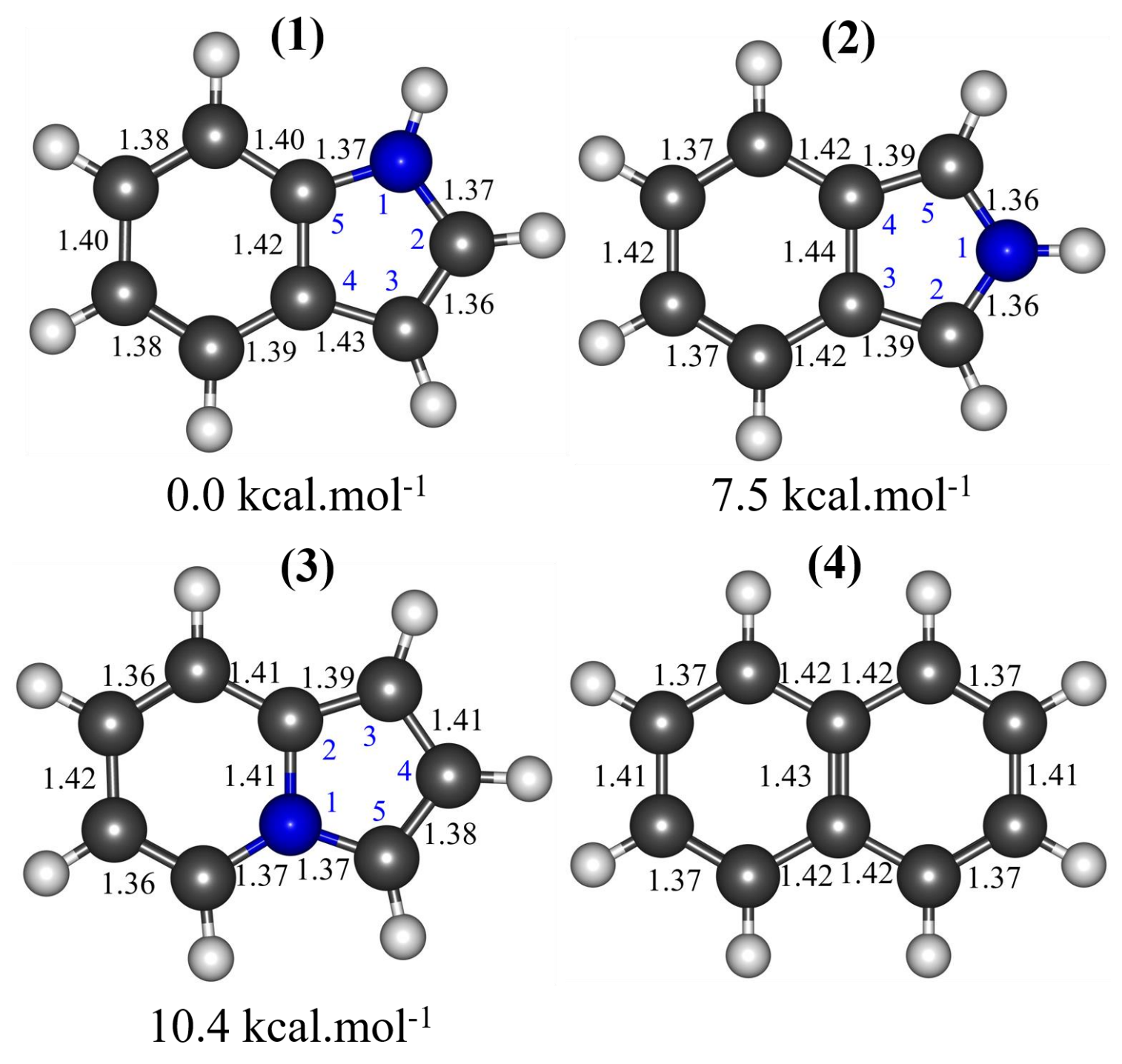

Figure 1. Molecular structures, atom numeration for the 5MR (in blue) and C-C, C-N bond lengths (black, in $\AA$ ) for Indole (1), Isoindole (2), Indolizine (3) and Naphthalene (4) optimized at the PBE0/6-

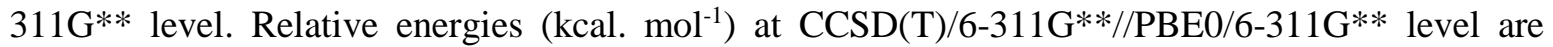
shown.

The magnetically induced current density of indole (Fig. 2a) presents a homogeneous diatropic (clockwise) current where local ones cannot be distinguished, in a similar way to the diatropic current presented by naphthalene (Fig 2d). If one considers that the intensity of the ring current correctly describes the aromatic character of a ring, this result is an indication that both rings that conform the 
bicyclic compound $\mathbf{1}$ present similar degrees of aromaticity. One way to confirm this result is through the quantification of the current flow through ring current strength calculations (RCS) measured in the ring junction. Table 1 shows that the RCS value between the 6MR in naphthalene is zero, while for the case of $\mathbf{1}$, this difference is only $1.0 \mathrm{nA}^{-\mathrm{T}^{-1}}$, the positive value denotes that the intensity of the ring current of the $6 \mathrm{MR}$ is slightly more intense than that of the 5MR. It is important to notice that this difference is only $0.5 \mathrm{nA} . \mathrm{T}^{-1}$ greater than the difference between the RCS values of the free rings of benzene and pyrrole, showing that aromaticity of the free rings is maintained in the local fused rings of 1. On the other hand, the isomers present significant differences in the diatropic current distribution, and homogeneity has been lost allowing to observe a local diatropic current in the 5MR of $\mathbf{2}$ and more noticeable in $\mathbf{3}$. When we examine the RCS results in the shared bond, they present values of -4.4 and $-6.1 \mathrm{nA} . \mathrm{T}^{-1}$ for $\mathbf{2}$ and $\mathbf{3}$ respectively, which indicates that 5MR presents greater aromaticity with respect to $6 \mathrm{MR}$. Fig. S1 presents the RCS values for all the ring bonds of the studied systems.As can be seen, the aromaticity of $6 \mathrm{MR}$ is reduced, while the aromaticity of the pyrrolic ring is increased, indicating a preference of the molecule to accentuate the local aromatic character of the smaller ring in agreement with the GLR (vide infra), when the isomers are ordered as follows: $\mathbf{1} \rightarrow \mathbf{2}$ $\rightarrow 3$. These results are confirmed through calculation of the popular $\operatorname{NICS}_{z z}(1)$ index (see Table 1), which is especially useful in studies of $\pi$-aromaticity in organic compounds. ${ }^{44-47}$ 
(1)

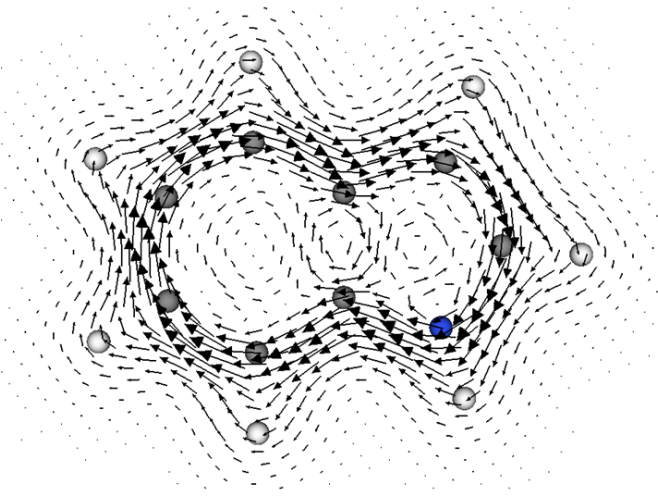

(3)

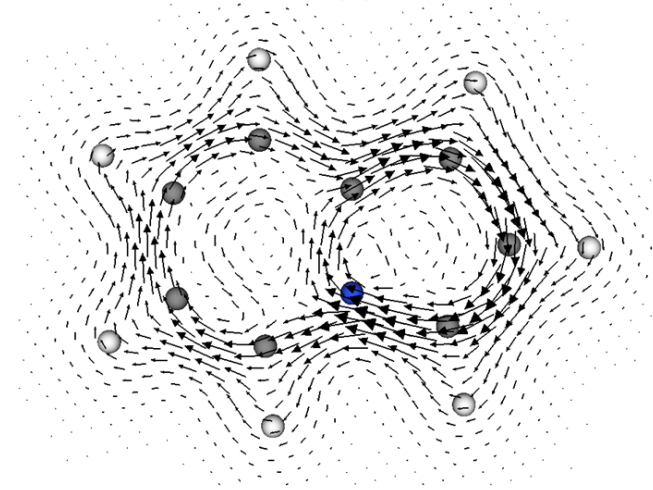

(2)

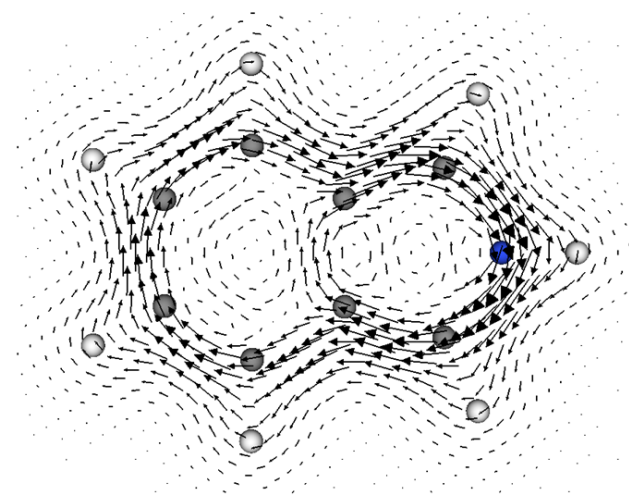

(4)

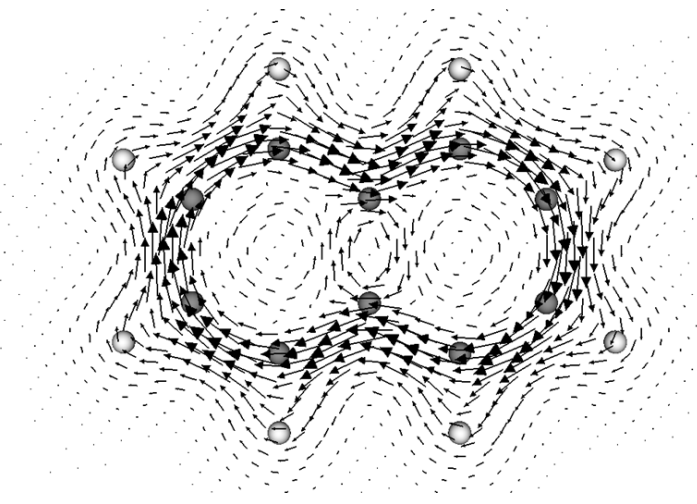

Figure 2. Magnetically induced current density vector maps for (1) Indole, (2) Isoindole, (3) Indolizine and (4) Naphthalene at the PBE0/6-311G** level.

To confirm the results obtained by means of the magnetic approach, geometrical and delocalization criteria have been applied for the characterization of the aromaticity of the local 6MR and 5MR rings (Table 1). According to the HOMA index, the 6MR of $\mathbf{1}$ is less aromatic than benzene, however, it has a higher aromaticity than that of naphthalene (4). The two isomers show a marked reduction in the aromaticity of the $6 \mathrm{MR}$ when compared to the $6 \mathrm{MR}$ of $\mathbf{1}$ and benzene. For the case of the 5MRs, the trend is opposite to that presented above, with a gradual increase of aromaticity from $\mathbf{1} \rightarrow \mathbf{3}$, the $5 \mathrm{MR}$ of $\mathbf{3}$ being as aromatic as pyrrole. Additionally, the energetic criteria, whose reference reactions can be seen in Figure S3, shows that 6MR in $\mathbf{1}$ is less aromatic than benzene but more aromatic than in 4. In addition, the 6MR in 2 and 3 present differences of only $1.5 \mathrm{kcal}^{\mathrm{mol}}{ }^{-1}$, showing lower aromatic character than in $\mathbf{1}$ in agreement with the shown above. For 5MRs, the aromaticity is lower 
in $\mathbf{1}$ than in $\mathbf{3}$, however 5MR in $\mathbf{2}$ has a very low ISE value. The reference reactions for the rings have been selected so they will affect the adjacent ring the least. However, for $\mathbf{2}$, it is not possible to design an isomerization reaction for 5MR that does not affect the aromaticity of 6MR (See Fig S4 in SI).

Furthermore, the delocalization criteria on the basis of MCI and PDI indexes show similar results to those previously shown. The values of MCI for the 6MR (six-center-delocalization) and PDI show a similar trend to the magnetic criterion, with a continuous reduction of aromaticity from $\mathbf{1} \rightarrow \mathbf{3}$. The $6 \mathrm{MRs}$ of naphthalene are more aromatic than those of $\mathbf{2}$ and $\mathbf{3}$ but less than that of $\mathbf{1}$. With respect to the 5MR, MCI (five-center-delocalization) results indicate that these rings in $\mathbf{1}$ and its isomers are less aromatic than pyrrole, with a tendency to increase in the order $\mathbf{1} \rightarrow \mathbf{3}$. The results found by the geometric and delocalization criteria confirm the results obtained by the magnetic criteria, showing that there is a reduction in aromaticity at the 6MR and a tendency for the 5MR to increase when going from 1 to 3 .

Table 1. RCS (in nA.T ${ }^{-1}$ ), NICS zz $_{\text {(1) }}$ (in ppm), HOMA, ISE (in kcal.mol ${ }^{-1}$ ), MCI (in a.u.) and PDI (in a.u.) results at the $\mathrm{PBE} 0 / 6-311 \mathrm{G}^{* *}$.

\begin{tabular}{cccccccccccc}
\hline \multirow{2}{*}{ Compound } & RCS & \multicolumn{2}{c}{ NICS $_{\text {zz }}(\mathbf{1})$} & \multicolumn{2}{c}{ HOMA } & \multicolumn{2}{c}{ ISE } & \multicolumn{3}{c}{ MCI } & PDI \\
\cline { 2 - 10 } & & Shared bond & $\mathbf{6 M R}$ & $\mathbf{5 M R}$ & $\mathbf{6 M R}$ & $\mathbf{5 M R}$ & $\mathbf{6 M R}$ & $\mathbf{5 M R}$ & $\mathbf{6 M R}$ & $\mathbf{5 M R}$ & $\mathbf{6 M R}$ \\
\hline Indole & 1.0 & -30.4 & -29.8 & 0.9415 & 0.7781 & 28.8 & 18.7 & 0.0523 & 0.0424 & 0.0826 \\
Isoindole & -4.4 & -23.5 & -38.8 & 0.7028 & 0.8177 & 19.6 & 8.1 & 0.0350 & 0.0546 & 0.0689 \\
Indolizine & -6.1 & -18.5 & -39.8 & 0.7588 & 0.8658 & 21.1 & 28.8 & 0.0244 & 0.0553 & 0.0624 \\
Naphthalene & 0.0 & -29.7 & - & 0.8262 & - & 25.2 & - & 0.0449 & - & 0.0765 \\
Pyrrole & $11.5^{\mathrm{a}}$ & - & -31.7 & - & 0.8883 & - & 23.2 & - & 0.0759 & - \\
Benzene & $12.0^{\mathrm{a}}$ & -29.8 & - & 0.9982 & - & 34.5 & - & 0.0774 & - & 0.1052 \\
\hline
\end{tabular}

${ }^{\text {a}}$ Value obtained by averaging the RCS values of all bonds in the ring. 
According to the GLR, among the $10 \pi$-electrons of the $\pi$-system of indole, isoindole, and indolizine, there is a preference to locate $6 \pi$-electrons in the $5 \mathrm{MR}$ (the smallest ring) and $4 \pi$-electrons in the 6MR. To determine the Kekulé structures that most contribute to the understanding of the electronic properties of $\mathbf{1}$ and its isomers, NRT calculations have been carried out. The results are shown in Fig. 3 and indicate that for $\mathbf{1}$, the structure that contributes most $(27 \%)$ is the one where the aromatic structures of the free 6MR and 5MR persist in the bicyclic compound (see Fig. S2 in SI) sharing a $\pi$ bond. Additionally, the second most important Kekulé structure (22\%) contains a $\pi$-sextet in the 6MR. Interestingly, $\mathbf{1}$ is the only system in which the covalent resonant structure with the largest contribution locates a $\pi$-bond in the ring junction between the 5- and 6-MRs. In this resonant structure, the two rings share $2 \pi$-electrons and, consequently, one can consider that both rings have $6 \pi$-electrons accounting for the almost equalization of the bonds that conform the bicyclic compound. This is why both rings have relatively large aromaticity and this explains the larger stability of $\mathbf{1}$ with respect to $\mathbf{2}$ and 3. For both $\mathbf{2}$ and $\mathbf{3}$, the most contributing structure is that containing $6 \pi$-electrons in the $5 \mathrm{MR}$, with the 6MR presenting a conjugation reminiscent of a 1,3-cyclohexadiene for $\mathbf{2}$ and $\mathbf{3}$ (see bond lengths in Fig. 1). As a result, the aromaticity of the 5MR increases while that of the 6MR decreases. The second most important structures of $\mathbf{2}$ and $\mathbf{3}$ are of ionic type. In the case of $\mathbf{3}$, the 5MR in this second more relevant Kekulé structure has the same structure to that of pyrrole (see Figs. 3 and S2), while the 6MR has a distribution reminiscent of 1,4-cyclohexadiene. Results are consistent with the increased aromaticity of 5MR in $\mathbf{3}$. As indicated by the results obtained for the indole isomers, the GLR is manifested by the preservation of the aromatic character of 5MR and the reduction of the aromaticity of $6 \mathrm{MR}$. 
(a)

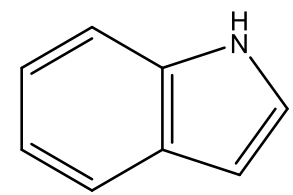

$27.1 \%$

(b)

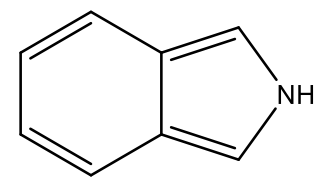

$18.3 \%$

(c)<smiles>c1ccn2cccc2c1</smiles>

$20.5 \%$

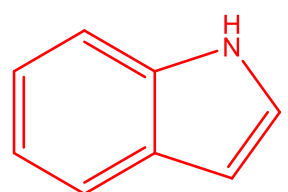

$22.0 \%$

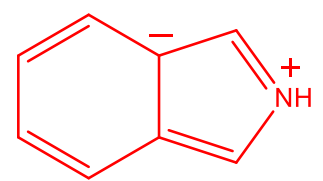

$14.3 \%$

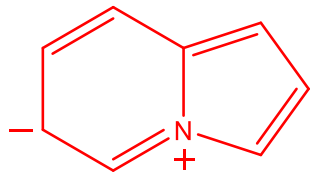

$13.5 \%$

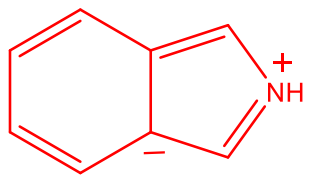

$14.2 \%$

Figure 3. Most important Kekulé structures according to Natural Resonance Theory (NRT) for (a) Indole, (b) Isoindole and (c) Indolizine at the PBE0/6-311G** level.

\section{Conclusions}

An analysis of the aromaticity of local rings of indole (1) and two of its isomers (isoindole (2) and indolizine (3)) has been carried out. All criteria used indicate a reduction of aromaticity in the 6MR through the following order: $\mathbf{1} \rightarrow \mathbf{2} \rightarrow \mathbf{3}$. The magnetic criterion manifests these differences through changes in the diatropic current of the 5- and 6-MRs. It is possible to measure these differences through ring current strength (RCS) calculations in the ring junction, obtaining values up to $-6.1 \mathrm{nA} . \mathrm{T}^{-}$ ${ }^{1}$ for the case of 3 , which suggests that in this conpound the 5MR ring is more aromatic than the $6 \mathrm{MR}$. These results are supported by $\operatorname{NICS}_{\mathrm{zz}}(1)$ measurements and the application of the geometrical and delocalization criteria through the HOMA and MCI and PDI indices. These indices show a marked reduction in aromaticity of $6 \mathrm{MR}$ and an slight increase of aromaticity in 5MR. In the case of isomerization reactions, although they show the same trends as the other indicators, caution must be taken in selecting the appropriate reference reaction. Results suggests that, in all cases, the aromaticity 
of 5MR is preserved over that of the 6MR. To confirm this, the Kekulé structures that contribute most to the electronic properties of these systems were obtained through NRT calculations showing that the structures in which 5MR retains its pyrrolic nature are the ones that contribute the most. These results lead us to conclude that the lower stability of isoindole and indolizine is the result of the reduction of the 6MR aromaticity as a manifestation of the Glidewell-Lloyd rule.

\section{Acknowledgements}

R.P-R. is grateful for the financial support of the grant: FONDECYT Postdoctorado 3180119. Powered@NLHPC. This research was partially supported by the supercomputing infrastructure of the NLHPC (ECM-02). M.S. acknowledges the financial support from the Ministerio de Economía y Competitividad of Spain (project CTQ2017-85341-P) and the Generalitat de Catalunya (project 2017SGR39). This work is dedicated to Prof. Alexander Boldyrev as a proof of our admiration for their brilliant contributions to chemistry. 


\section{Supplementary Information}

Detailed RCS values (in nA. $\mathrm{T}^{-1}$ ), Selected and discarded isomerization reactions, Natural Resonance

Theory results for benzene and pyrrole and cartesian coordinates of $\mathbf{1}-\mathbf{4}$.

\section{References}

1. Mohammadi Ziarani, G.; Moradi, R.; Ahmadi, T.; Lashgari, N., Recent advances in the application of indoles in multicomponent reactions. RSC Adv. 2018, 8, 12069-12103.

2. $\quad$ Festa, A. A.; Voskressensky, L. G.; Van der Eycken, E. V., Visible light-mediated chemistry of indoles and related heterocycles. Chem. Soc. Rev. 2019, 48, 4401-4423.

3. $\quad$ Sundberg, R., The Chemistry of Indoles. Elsevier Science: 2012.

4. Joule, J. A.; Mills, K., Heterocyclic Chemistry. Wiley: 2013.

5. Dewar, J. S.; Harget, A. J.; Trinajstić, N.; Worley, S. D., Ground states of conjugated molecules-XXI: Benzofurans and benzopyrroles. Tetrahedron 1970, 26, 4505-4516.

6. $\quad$ Chacko, E.; Bornstein, J.; Sardella, D. J., Aromaticity and chemical reactivity in isoindole. Tetrahedron Lett. 1977, 18, 1095-1098.

7. Mandado, M.; Otero, N.; Mosquera, R. A., Local aromaticity study of heterocycles using ncenter delocalization indices: the role of aromaticity on the relative stability of position isomers. Tetrahedron 2006, 62, 12204-12210.

8. $\quad$ Clar, E., The aromatic sextet. Wiley: New York, 1972.

9. Solà, M., Forty years of Clar's aromatic $\pi$-sextet rule. Front Chem 2013, 1, 22.

10. Glidewell, C.; Lloyd, D., Mndo study of bond orders in some conjugated BI- and tri-cyclic hydrocarbons. Tetrahedron 1984, 40, 4455-4472.

11. El Bakouri, O.; Poater, J.; Feixas, F.; Solà, M., Exploring the validity of the Glidewell-Lloyd extension of Clar's $\pi$-sextet rule: assessment from polycyclic conjugated hydrocarbons. Theor. Chem. Acc. 2016, 135, 205.

12. Poater, J.; García-Cruz, I.; Illas, F.; Solà, M., Discrepancy between common local aromaticity measures in a series of carbazole derivatives. Phys. Chem. Chem. Phys. 2004, 6, 314-318.

13. Báez-Grez, R.; Pino-Rios, R., Borataalkene or boratabenzene? Understanding the aromaticity of 9-borataphenanthrene anions and its central ring. New J. Chem. 2020, 44, 18069-18073.

14. Glendening, E. D.; Weinhold, F., Natural resonance theory: I. General formalism. J. Comput. Chem. 1998, 19, 593-609.

15. Glendening, E. D.; Weinhold, F., Natural resonance theory: II. Natural bond order and valency. J. Comput. Chem. 1998, 19, 610-627.

16. Glendening, E. D.; Badenhoop, J. K.; Weinhold, F., Natural resonance theory: III. Chemical applications. J. Comput. Chem. 1998, 19, 628-646.

17. Hehre, W. J.; Ditchfield, R.; Pople, J. A., Self - Consistent Molecular Orbital Methods. XII. Further Extensions of Gaussian-Type Basis Sets for Use in Molecular Orbital Studies of Organic Molecules. J. Chem. Phys. 1972, 56, 2257-2261.

18. Ditchfield, R.; Hehre, W. J.; Pople, J. A., Self-Consistent Molecular-Orbital Methods. IX. An Extended Gaussian-Type Basis for Molecular-Orbital Studies of Organic Molecules. J. Chem. Phys. 1971, 54, 724-728.

19. Adamo, C.; Barone, V., Toward reliable density functional methods without adjustable parameters: The PBE0 model. J. Chem. Phys. 1999, 110, 6158-6170.

20. Frisch, G. W.; Schlegel, H. B.; Scuseria, G. E.; Robb, M. A.; Cheeseman, J. R.; Scalmani, G.; Barone, V.; Petersson, G. A.; Nakatsuji, H.; Li, X.; et al., Gaussian 16, Rev. B.01. Gaussian, Inc., Wallingford, CT 2016. 
21. Bartlett, R. J.; Musiał, M., Coupled-cluster theory in quantum chemistry. Rev. Mod. Phys. 2007, 79, 291-352.

22. Gershoni-Poranne, R.; Stanger, A., Magnetic criteria of aromaticity. Chem. Soc. Rev. 2015, 44, 6597-615.

23. Wolinski, K.; Hinton, J. F.; Pulay, P., Efficient implementation of the gauge-independent atomic orbital method for NMR chemical shift calculations. J Am Chem Soc 1990, 112, 8251-8260.

24. Sundholm, D.; Fliegl, H.; Berger, R. J. F., Calculations of magnetically induced current densities: theory and applications. WIREs Computational Molecular Science 2016, 6, 639-678.

25. Bader, R. F. W., Atoms in Molecules: A Quantum Theory. Clarendon Press: Oxford, 1994.

26. Keith, T. A., AIMAll (Version 19.02.13). Overland Park KS, USA, 2019 (aim.tkgristmill.com).

27. Keith, T. A., Calculation of magnetizabilities using GIAO current density distributions. Chem. Phys. 1996, 213, 123-132.

28. Keith, T. A.; Bader, R. F. W., Calculation of magnetic response properties using a continuous set of gauge transformations. Chem. Phys. Lett. 1993, 210, 223-231.

29. Keith, T. A.; Bader, R. F. W., Calculation of magnetic response properties using atoms in molecules. Chem. Phys. Lett. 1992, 194, 1-8.

30. Schleyer, P. v. R.; Jiao, H.; Hommes, N. J. R. v. E.; Malkin, V. G.; Malkina, O. L., An Evaluation of the Aromaticity of Inorganic Rings: Refined Evidence from Magnetic Properties. $J$. Am. Chem. Soc. 1997, 119, 12669-12670.

31. Cernusak, I.; Fowler, P. W.; Steiner, E., Ring currents in six-membered heterocycles: the diazaborinines $(\mathrm{CH})_{2} \mathrm{~B}_{2} \mathrm{~N}_{2}$. Mol. Phys. 2000, 98, 945-953.

32. Fowler, P. W.; Steiner, E.; Zanasi, R.; Cadioli, B., Electric and magnetic properties of hexaethynylbenzene. Mol. Phys. 1999, 96, 1099-1108.

33. Kruszewski, J.; Krygowski, T. M., Definition of aromaticity basing on the harmonic oscillator model. Tetrahedron Lett. 1972, 13, 3839-3842.

34. Krygowski, T. M.; Cyranski, M. K., Structural aspects of aromaticity. Chem. Rev. 2001, 101, 1385-419.

35. Schleyer, P. v. R.; Pühlhofer, F., Recommendations for the Evaluation of Aromatic Stabilization Energies. Org. Lett. 2002, 4, 2873-2876.

36. Poater, J.; Duran, M.; Solà, M.; Silvi, B., Theoretical Evaluation of Electron Delocalization in Aromatic Molecules by Means of Atoms in Molecules (AIM) and Electron Localization Function (ELF) Topological Approaches. Chem. Rev. 2005, 105, 3911-3947.

37. Feixas, F.; Matito, E.; Poater, J.; Solà, M., Quantifying aromaticity with electron delocalisation measures. Chem. Soc. Rev. 2015, 44, 6434-51.

38. Giambiagi, M.; de Giambiagi, M. S.; Mundim, K. C., Definition of a multicenter bond index. Struct. Chem. 1990, 1, 423-427.

39. Poater, J.; Fradera, X.; Duran, M.; Solà, M., The delocalization index as an electronic aromaticity criterion: application to a series of planar polycyclic aromatic hydrocarbons. Chem. Eur. J. 2003, 9, 400-6.

40. Lu, T.; Chen, F., Multiwfn: a multifunctional wavefunction analyzer. J. Comput. Chem. 2012, 33, 580-92.

41. Glendening, E. D.; Landis, C. R.; Weinhold, F., NBO 6.0: Natural bond orbital analysis program. J. Comput. Chem. 2013, 34, 1429-1437.

42. Childs, H.; Brugger, E.; Whitlock, B.; Meredith, J.; Ahern, S.; Pugmire, D.; Biagas, K.; Miller, M.; Harrison, C.; Weber, G.; et al. VisIt: An end-user tool for visualizing and analyzing very large data. In High Performance Visualization-Enabling Extreme-Scale Scientific Insight. Chapman and Hall/CRC: Boca Ratón, 2012, pp. 357-372.

43. Chemcraft - graphical software for visualization of quantum chemistry computations. (https://www.chemcraftprog.com. 
44. Islas, R.; Heine, T.; Merino, G., The induced magnetic field. Acc. Chem. Res. 2012, 45, 21528.

45. Merino, G.; Heine, T.; Seifert, G., The induced magnetic field in cyclic molecules. Chem. Eur. J. 2004, 10, 4367-71.

46. Pino-Rios, R.; Cardenas-Jiron, G.; Ruiz, L.; Tiznado, W., Interpreting Aromaticity and Antiaromaticity through Bifurcation Analysis of the Induced Magnetic Field. ChemistryOpen 2019, 8, 321-326.

47. Báez-Grez, R.; Ruiz, L.; Pino-Rios, R.; Tiznado, W., Which NICS method is most consistent with ring current analysis? Assessment in simple monocycles. RSC Adv. 2018, 8, 13446-13453. 


\section{Table of Contents}

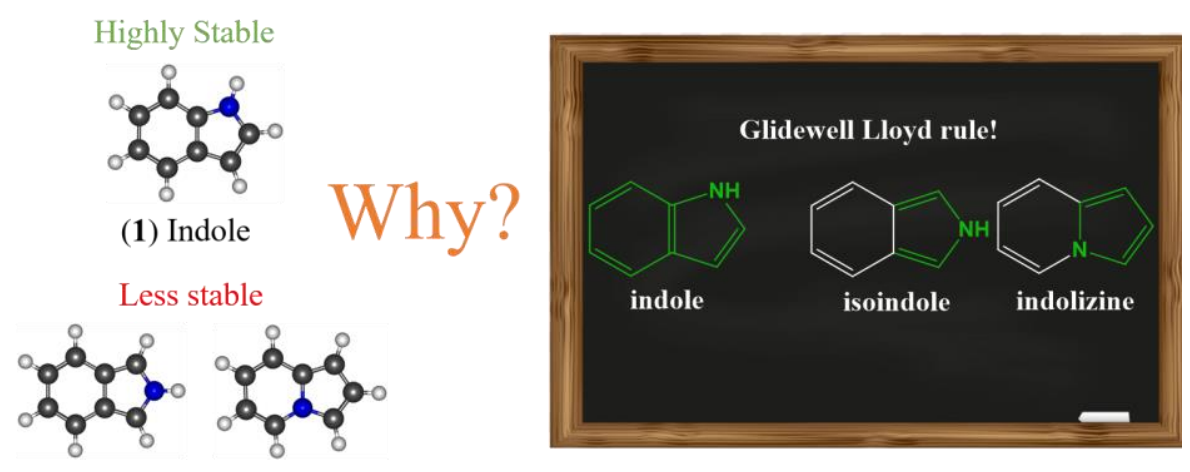

(2) Isoindole (3) Indolizine

The difference in stability between indole and its isomers is explained in terms of local aromaticity.

Diverse criteria used in this work indicate that the aromaticity of the six-membered ring is reduced when going from $\mathbf{1}$ to $\mathbf{3}$ whereas in the five-membered ring is retained, as a consequence of the Glidewell-Lloyd rule. 